Document downloaded from:

http://hdl.handle.net/10251/125657

This paper must be cited as:

Burgos-Simon, C.; Calatayud-Gregori, J.; Cortés, J.; Navarro-Quiles, A. (2018). A full probabilistic solution of the random linear fractional differential equation via the Random Variable Transformation technique. Mathematical Methods in the Applied Sciences. 41(18):9037-9047. https://doi.org/10.1002/mma.4881

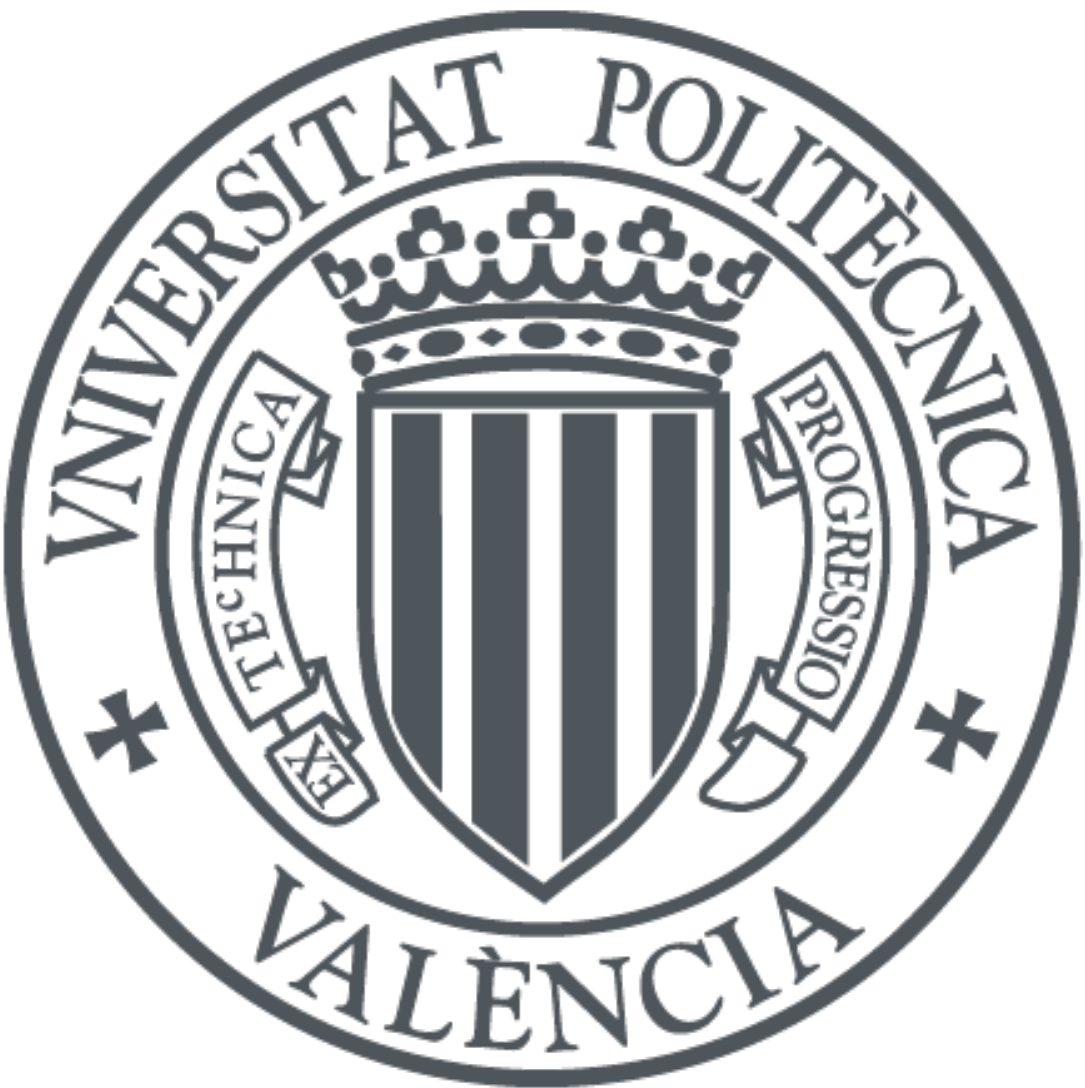

The final publication is available at

http://doi.org/10.1002/mma.4881

Copyright John Wiley \& Sons

Additional Information 


\title{
A full probabilistic solution of the random linear fractional differential equation via the Random Variable Transformation technique
}

\author{
C. Burgos ${ }^{a}$, J. Calatayud ${ }^{a}$, J.-C. Cortés ${ }^{a, *}$, A. Navarro-Quiles ${ }^{a}$
}

This paper provides a full probabilistic solution of the randomized fractional linear non-homogeneous differential equation with a random initial condition via the computation of the first probability density function of the solution stochastic process. To account for most generality in our analysis, we assume that uncertainty appears in all input parameters (diffusion coefficient, source term and initial condition) and that a wide range of probabilistic distributions can be assigned to these parameters. Throughout our study we will consider that the fractional order of Caputo derivative lies in ]0, 1], that corresponds to the main standard case. To conduct our analysis, we take advantage of the Random Variable Transformation technique to construct approximations of the first probability density function of the solution process from a suitable infinite series representation. We then prove these approximations do converge to the exact density assuming mild conditions on random input parameters. Our theoretical findings are illustrated through two numerical examples.

Keywords: Random fractional differential equations; Random Variable Transformation technique; First probability density function.

\section{Introduction and motivation}

Over the last decades, fractional differential equations are having an impact in mathematical modelling in a variety of complex problems belonging to different scientific areas. Apart from the intrinsec interest of Fractional Calculus as an extension of the Newton-Leibniz Calculus, hence providing smart generalizations of classical results [1, 2], a number of contributions has shown its potentiality to model problems where memory plays a key role into the modelling process. Specific examples can be found in different realms, for example in Engineering where appear problems of viscoelasticity, electromagnetism, etc., whose answers depend upon memory and hereditary properties of materials [3, 4]; in Epidemiology, where competition dynamics may reinforce certain genogroups by DNA recombination or mutations and this would depend on the other genogroups coexisting with them as well as the time this coexistence lasts and their populations [5].

On the one hand, the aforementioned strong impact of fractional differential equations in mathematical modelling applications, and on the other hand, the need of quantifying uncertainty involved in measurements or surveys used to fix the input parameters of fractional differential equations lead to two main classes of fractional differential equations with uncertainty, namely, Stochastic Fractional Differential Equations (SFDEs) and Random Fractional Differential Equations (RFDEs). A comprehensive overview on the ways that uncertainty can be considered in fractional differential equations can be found in [6]. It is important to point out that besides solving SFDEs and RFDEs, in the stochastic/random context a major goal is to compute the main statisitical information associated to the solution as well. In the context of SFDEs, uncertainty is introduced via stochastic processes, like Wiener process, having an irregular sample behaviour (nowhere differentiable). The analytic and numerical treatment of SDEs requires the socalled Itô-Doob Calculus [7, 8, 9]. Some interesting applications based on this approach are reported in [10, 11, 12], for instance. While RFDEs consist of the direct randomization of input parameters of their deterministic counterpart via random variables or regular stochastic processes (e.g., sample continuous). The analysis of RFDEs has been undertaken recently. Interesting results

a Instituto Universitario de Matemática Multidisciplinar

Universitat Politècnica de València,

Camino de Vera s/n, 46022, Valencia, Spain

* Correspondence to: J.-C. Cortés, Instituto Universitario de Matemática Multidisciplinar, Universitat Politècnica de València, Camino de Vera s/n, 46022, Valencia, Spain

†E-mail: clabursi@doctor.upv.es; jucagre@alumni.uv.es; jccortes@imm.upv.es; annaqui@doctor.upv.es 
on the existence and uniqueness of solutions for initial value problems (IVPs) for random fractional differential equations that extend their deterministic counterpart are exhibited in $[13,14]$. In [15] one adapts the polynomial chaos method to solve some RFDEs. Recently, some of the authors have studied autonomous and non-autonomous linear RFDEs using the so-called mean square calculus $[16,17]$. In both contributions, we have taken advantage of random Fröbenius method to solve linear RFDEs through mean square random generalized power series and then to obtain the statistical information of the solution stochastic process through the mean and the variance. However, if $y(t, \omega)$ denotes the solution stochastic process of a RFDE defined in a complete probability space $(\Omega, \mathcal{F}, \mathbb{P})$, then a more ambitious target is the computation of the first probability density function (1-PDF), $f_{1}(y, t)$, of $y(t, \omega)$ because from it one can compute all one-dimensional statistical moments at every time instant $t$,

$$
\mathbb{E}\left[(y(t, \omega))^{k}\right]=\int_{-\infty}^{\infty}(y(t))^{k} f_{1}(y, t) \mathrm{d} y, \quad k=1,2, \ldots, \quad \omega \in \Omega .
$$

In particular, the mean $\mu_{y}(t)=\mathbb{E}[y(t, \omega)]$ and the variance $\sigma_{y}^{2}(t)=\mathbb{V}[y(t, \omega)]=\mathbb{E}\left[(y(t, \omega))^{2}\right]-\left(\mu_{y}(t)\right)^{2}$. Furthermore, the 1-PDF allows us to calculate the probability that the solution lies in a particular interval of interest at each time instant $\hat{t}$ fixed, i.e.,

$$
\mathbb{P}[a \leq y(\hat{t}, \omega) \leq b]=\int_{a}^{b} f_{1}(y, \hat{t}) \mathrm{d} y .
$$

In this paper, we shall go beyond and we revisit the linear RFDE in order to compute the 1-PDF of its solution stochastic process under mild conditions. Although the computation of the 1-PDF of different classes of random differential equations has been dealt in the extant literature (see $[18,19,20,21]$ and references therein), to the best of our knowledge, this interesting problem is not been addressed yet. In this spirit, let us consider the following random IVP

$$
\left.\begin{array}{cl}
\left({ }^{C} D_{0}^{\alpha} y\right)(t, \omega)-\lambda(\omega) y(t, \omega) & =\gamma(\omega), \quad 0<\alpha \leq 1, \quad t>0 \\
y(0, \omega) & =\beta_{0}(\omega),
\end{array}\right\}
$$

where ${ }^{C} D_{0}^{\alpha}$ stands for the random Caputo derivative defined in [13]. In (2), the initial condition $\beta_{0}(\omega)$, the forcing term $\gamma(\omega)$ and the diffusion coefficient $\lambda(\omega)$ are assumed to be absolutely continuous random variables defined on a common complete probability space $(\Omega, \mathcal{F}, \mathbb{P})$. For sake of clarity, hereinafter we will denote by $\mathcal{D}_{\mathbf{X}}$ the domain of any random vector, say $\mathbf{X}(\omega)$, in particular, $\mathcal{D}_{\left(\beta_{0}, \gamma, \lambda\right)}$ will stand for the domain of input random vector $\mathbf{X}(\omega)=\left(\beta_{0}(\omega), \gamma(\omega), \lambda(\omega)\right)$ and $f_{\beta_{0}, \gamma, \lambda}\left(\beta_{0}, \gamma, \lambda\right)$ will denote its joint PDF.

According to [16], using a random Fröbenius method, the solution stochastic process of random fractional IVP (2) can be represented by

$$
y(t, \omega)=\beta_{0}(\omega) S_{1}(t, \alpha ; \lambda(\omega))+\gamma(\omega) S_{2}(t, \alpha ; \lambda(\omega)),
$$

being

$$
S_{1}(t, \alpha ; \lambda(\omega))=\sum_{m=0}^{\infty} \frac{(\lambda(\omega))^{m}}{\Gamma(\alpha m+1)} t^{\alpha m}, \quad S_{2}(t, \alpha ; \lambda(\omega))=\sum_{m=0}^{\infty} \frac{(\lambda(\omega))^{m}}{\Gamma(\alpha(m+1)+1)} t^{\alpha(m+1)} .
$$

Remark 1.1 As it can be observed in (3)-(4) series, $S_{1}(t, \alpha ; \lambda(\omega))$ and $S_{2}(t, \alpha ; \lambda(\omega))$, and thus the solution $y(t, \omega)$, can be represented as a random series in both parameters $\lambda(\omega), \omega \in \Omega$, and $t$. Taking into account the uniqueness of the solution of random IVP (2), both series expansions match (as powers of $t$ and as powers of $\lambda(\omega), \omega \in \Omega$ ). In addition, it can be proved by applying the ratio test together with Stirling's formula that both series converge in $\mathbb{R}$ for all $\omega \in \Omega$, Therefore, there is uniform convergence in every closed set contained in $\mathbb{R}$.

As the solution $y(t, \omega)$ of random IVP (2) is given by a linear combination of infinite series (3)-(4), to compute its 1-PDF, $f_{1}(y, t)$, from a computational point of view it is convenient to consider its approximation via the truncation of both series

$$
y^{M}(t, \omega)=\beta_{0}(\omega) S_{1}^{M}(t, \alpha ; \lambda(\omega))+\gamma(\omega) S_{2}^{M}(t, \alpha ; \lambda(\omega))
$$

where

$$
S_{1}^{M}(t, \alpha ; \lambda(\omega))=\sum_{m=0}^{M} \frac{(\lambda(\omega))^{m}}{\Gamma(\alpha m+1)} t^{\alpha m}, \quad S_{2}^{M}(t, \alpha ; \lambda(\omega))=\sum_{m=0}^{M} \frac{(\lambda(\omega))^{m}}{\Gamma(\alpha(m+1)+1)} t^{\alpha(m+1)} .
$$

Then, we will first obtain the 1-PDF, $f_{1}^{M}(y, t)$, of the truncated solution $y^{M}(t, \omega)$, and second, we will prove that $f_{1}^{M}(y, t) \rightarrow$ $f_{1}(y, t)$ as $M \rightarrow \infty$, under certain conditions on the input random vector $\left(\beta_{0}(\omega), \gamma(\omega), \lambda(\omega)\right)$ that will be specified later.

To compute $f_{1}^{M}(y, t)$, Random Variable Transformation method (RVT) will be applied. This technique allows us to obtain the PDF of a random vector that results from mapping of another random vector whose PDF is known. For the sake of completeness in the presentation and notation, down below we state a multidimensional version of RVT method.

Theorem 1.2 (Multidimensional Random Variable Transformation method) [22, p.25]. Let us consider $\mathbf{X}=\left(X_{1}, \ldots, X_{n}\right)$ and $\mathbf{Z}=\left(Z_{1}, \ldots, Z_{n}\right)$ two $n$-dimensional absolutely continuous random vectors defined on a common probability space $(\Omega, \mathcal{F}, \mathbb{P})$. Let $\mathbf{r}: \mathbb{R}^{n} \rightarrow \mathbb{R}^{n}$ be a one-to-one deterministic transformation of $\mathbf{X}$ into $\mathbf{Z}$, i.e., $\mathbf{Z}=\mathbf{r}(\mathbf{X})$. Assume that $\mathbf{r}$ is continuous in 
$\mathbf{X}$ and has continuous partial derivatives with respect to each $X_{i}, 1 \leq i \leq n$. Then, if $f_{\mathbf{X}}(\mathbf{x})$ denotes the joint probability density function of random vector $\mathbf{X}$, and $\mathbf{s}=\mathbf{r}^{-1}=\left(s_{1}\left(z_{1}, \ldots, z_{n}\right), \ldots, s_{n}\left(z_{1}, \ldots, z_{n}\right)\right)$ represents the inverse mapping of $\mathbf{r}=\left(r_{1}\left(x_{1}, \ldots, x_{n}\right), \ldots, r_{n}\left(x_{1}, \ldots, x_{n}\right)\right)$, the joint probability density function of random vector $\mathbf{Z}$ is given by

$$
f_{\mathrm{Z}}(\mathbf{z})=f_{\mathrm{X}}(\mathbf{h}(\mathbf{z}))|J|
$$

where $|J|$, which is assumed to be different from zero, is the absolute value of the Jacobian defined by the determinant

$$
J=\operatorname{det}\left(\frac{\partial \mathbf{s}^{\top}}{\partial \mathbf{z}}\right)=\operatorname{det}\left(\begin{array}{ccc}
\frac{\partial s_{1}\left(z_{1}, \ldots, z_{n}\right)}{\partial z_{1}} & \cdots & \frac{\partial s_{n}\left(z_{1}, \ldots, z_{n}\right)}{\partial z_{1}} \\
\vdots & \ddots & \vdots \\
\frac{\partial s_{1}\left(z_{1}, \ldots, z_{n}\right)}{\partial z_{n}} & \cdots & \frac{\partial s_{n}\left(z_{1}, \ldots, z_{n}\right)}{\partial z_{n}}
\end{array}\right) .
$$

The following technical remark will be useful later to legitimate the application of RVT technique in an appropriate neighbourhood where the 1-PDF of the solution stochastic process will be computed.

Remark 1.3 Let $\alpha \in] 0,1]$, taking into account expression (6) it is clear that $S_{1}^{M}(0, \alpha ; \lambda(\omega))=1 \neq 0, \forall \omega \in \Omega, \forall M \geq 0$ integer. From Remark $1.1 S_{1}(t, \alpha ; \lambda(\omega))$ can be represented via a power series in $t$ and $\lambda(\omega), \forall \omega \in \Omega$. Since $S_{1}^{M}(t, \alpha ; \lambda(\omega))$ is a polynomial in both variables $t$ and $\lambda(\omega)$, then is a continuous function in these variables. Therefore, there exists a neighbourhood, $\mathcal{N}(0,0(\omega))$ of $(t=0, \lambda(\omega)=0(\omega))$ such that

$$
0<m_{S_{1}}<\left|S_{1}^{M}(t, \alpha ; \lambda(\omega))\right|, \quad \forall(t, \lambda(\omega)) \in \mathcal{N}(0,0(\omega)), \quad \forall \omega \in \Omega, \quad \forall M \geq 0 \text { integer. }
$$

On the other hand, by Remark 1.1 both series $S_{i}(t, \alpha ; \lambda(\omega)), i=1,2$, converge uniformly in every closed set of $\mathbb{R}$. Then, we can take, without loss of generality, the closure of neighbourhood $\mathcal{N}(0,0(\omega)), \mathcal{N}^{*}(0,0(\omega))$. This guarantees the existence of positive constants $M_{S_{i}}, i=1,2$, such that

$$
\left|S_{i}^{M}(t, \alpha ; \lambda(\omega))\right|<M_{S_{i}}, \quad \forall(t, \lambda(\omega)) \in \mathcal{N}^{*}(0,0(\omega)) \subset \mathcal{N}(0,0(\omega)), \quad \forall \omega \in \Omega, \quad \forall M \geq 0, \text { integer, } \quad i=1,2 .
$$

Notice that in Remark 1.3, we have taken the neighbourhood of point $(t, \lambda(\omega))=(0,0(\omega))$. This choice is motivated by the expression of $S_{1}(t, \alpha ; \lambda(\omega))$ given in (4), where, as it has been previously pointed, one can observe that is a power series in $\lambda(\omega), \forall \omega \in \Omega$. Then, it is centered in $\lambda(\omega)=0(\omega), \forall \omega \in \Omega$.

This paper is organized as follows. In Section 2 the 1-PDF of the truncated solution stochastic process given by (5) is constructed by applying the RVT technique stated in Theorem 1.2. Then, we prove the convergence of this function to the 1-PDF of the exact solution of RFDE (2) under suitable hypotheses on random inputs. Section 3 is devoted to show several examples that illustrate our theoretical findings. Finally, in Section 4 our main conclusions are drawn.

\section{Main result: Computing approximations of the 1-PDF of the solution stochastic process}

In this section, we will first compute the 1-PDF $f_{1}^{M}(y, t)$ by taking advantage of RVT technique. With this goal, let $\left.\left.\alpha \in\right] 0,1\right]$, $t>0, \omega \in \Omega$ fixed, such that $(t, \lambda(\omega))$ belongs to the neighbourhood $\mathcal{N}^{*}(0,0(\omega))$ introduced in Remark 1.3 and let us apply Theorem 1.2 with the following identification: $\mathbf{X}(\omega)=\left(\beta_{0}(\omega), \gamma(\omega), \lambda(\omega)\right), \mathbf{Z}(\omega)=\left(Z_{1}(\omega), Z_{2}(\omega), Z_{3}(\omega)\right)$ and the mapping $\mathbf{r}: \mathbb{R}^{3} \longrightarrow \mathbb{R}^{3}$ whose components $\left(r_{1}, r_{2}, r_{3}\right)$ are defined as

$$
\begin{aligned}
& z_{1}=r_{1}\left(\beta_{0}, \gamma, \lambda\right)=\beta_{0} S_{1}^{M}(t, \alpha ; \lambda)+\gamma S_{2}^{M}(t, \alpha ; \lambda) \\
& z_{2}=r_{2}\left(\beta_{0}, \gamma, \lambda\right)=\gamma \\
& z_{3}=r_{3}\left(\beta_{0}, \gamma, \lambda\right)=\lambda
\end{aligned}
$$

The inverse mapping $\mathbf{s}$ of $\mathbf{r}$ is given by

$$
\begin{aligned}
& \beta_{0}=s_{1}\left(z_{1}, z_{2}, z_{3}\right)=\frac{z_{1}-z_{2} S_{2}^{M}\left(t, \alpha ; z_{3}\right)}{S_{1}^{M}\left(t, \alpha ; z_{3}\right)} \\
& \gamma=s_{2}\left(z_{1}, z_{2}, z_{3}\right)=z_{2}, \\
& \lambda=s_{3}\left(z_{1}, z_{2}, z_{3}\right)=z_{3},
\end{aligned}
$$

From (9), it is known that $\left|S_{1}^{M}(t, \alpha ; \lambda(\omega))\right| \neq 0, \forall(t, \lambda(\omega)) \in \mathcal{N}^{*}(0,0(\omega))$, and thus the absolute value of the Jacobian of inverse mapping $\mathbf{s}$ is well-defined and non-zero

$$
|J|=\left|\frac{\partial s_{1}}{\partial z_{1}}\right|=\left|\frac{1}{S_{1}^{M}\left(t, \alpha ; z_{3}\right)}\right| \neq 0 .
$$


As a consequence, by applying Theorem 1.2, the PDF of random vector $\mathbf{Z}(\omega)$ is given by

$$
f_{z_{1}, z_{2}, z_{3}}\left(z_{1}, z_{2}, z_{3}\right)=f_{\beta_{0}, \gamma, \lambda}\left(\frac{z_{1}-z_{2} S_{2}^{M}\left(t, \alpha ; z_{3}\right)}{S_{1}^{M}\left(t, \alpha ; z_{3}\right)}, z_{2}, z_{3}\right)\left|\frac{1}{S_{1}^{M}\left(t, \alpha ; z_{3}\right)}\right|
$$

Finally, marginalizing with respect to $Z_{2}(\omega)=\gamma(\omega)$ and $Z_{3}(\omega)=\lambda(\omega)$ and taking $t>0$ arbitrary, the 1-PDF of the truncated solution stochastic process, $y^{M}(t, \omega)$, of random fractional IVP (2) is obtained

$$
f_{1}^{M}(y, t)=\int_{\mathcal{D}(\gamma, \lambda)} f_{\beta_{0}, \gamma, \lambda}\left(\frac{y-\gamma S_{2}^{M}(t, \alpha ; \lambda)}{S_{1}^{M}(t, \alpha ; \lambda)}, \gamma, \lambda\right)\left|\frac{1}{S_{1}^{M}(t, \alpha ; \lambda)}\right| \mathrm{d} \lambda \mathrm{d} \gamma
$$

Now, we address the proof of the convergence of the 1-PDF, $f_{1}^{M}(y, t)$, given in (11), to the 1-PDF of the exact solution, $f_{1}(y, t)$, given by

$$
f_{1}(y, t)=\int_{\mathcal{D}(\gamma, \lambda)} f_{\beta_{0}, \gamma, \lambda}\left(\frac{y-\gamma S_{2}(t, \alpha ; \lambda)}{S_{1}(t, \alpha ; \lambda)}, \gamma, \lambda\right)\left|\frac{1}{S_{1}(t, \alpha ; \lambda)}\right| \mathrm{d} \lambda \mathrm{d} \gamma
$$

where $S_{1}(t, \alpha ; \lambda)$ and $S_{2}(t, \alpha ; \lambda)$ are defined in $(4)$, for each $(y, t) \in \mathbb{R} \times\left[0, \infty\left[\right.\right.$ fixed, as $M \rightarrow \infty$, i.e., $\lim _{M \rightarrow \infty} f_{1}^{M}(y, t)=$ $f_{1}(y, t)$ for each $(y, t) \in \mathbb{R} \times\left[0, \infty\right.$ [ fixed with $t$ such that $(t, \lambda(\omega)) \in \mathcal{N}^{*}(0,0(\omega))$. To this end, henceforth the following hypotheses will be assumed

H1: Inputs parameters $\beta_{0}(\omega), \gamma(\omega)$ and $\lambda(\omega)$ of random IVP (2) are independent random variables defined in a common complete probability space $(\Omega, \mathcal{F}, \mathbb{P})$.

H2: The expectation of the absolute value of random variable defining the non-homogeneous term of random IVP (2) is finite, i.e., $\mathbb{E}[|\gamma(\omega)|]=C<\infty$.

H3: The PDF of the random initial condition $f_{\beta_{0}}\left(\beta_{0}\right)$ is Lipschitz continuous in $\mathbb{R}$, i.e.,

$$
\exists L_{0}>0: \quad\left|f_{\beta_{0}}\left(\beta_{0,1}\right)-f_{\beta_{0}}\left(\beta_{0,2}\right)\right| \leq L_{0}\left|\beta_{0,1}-\beta_{0,2}\right|, \quad \forall \beta_{0,1}, \beta_{0,2} \in \mathbb{R}
$$

Now, we comment about the generality of above assumptions from a practical point of view. Hypothesis $\mathbf{H} \mathbf{1}$ is just imposed to simplify the subsequent development. In particular, it allows us to apply the following factorization $f_{\beta_{0}, \gamma, \lambda}\left(\beta_{0}, \gamma, \lambda\right)=$ $f_{\beta_{0}}\left(\beta_{0}\right) f_{\gamma}(\gamma) f_{\lambda}(\lambda)$. As a consequence, expression (11) can be written as follows

$$
f_{1}^{M}(y, t)=\int_{\mathcal{D}(\gamma)} \int_{\mathcal{D}(\lambda)} f_{\beta_{0}}\left(\frac{y-\gamma S_{2}^{M}(t, \alpha ; \lambda)}{S_{1}^{M}(t, \alpha ; \lambda)}\right) f_{\gamma}(\gamma) f_{\lambda}(\lambda)\left|\frac{1}{S_{1}^{M}(t, \alpha ; \lambda)}\right| \mathrm{d} \lambda \mathrm{d} \gamma
$$

Independence among random inputs is a usual assumption in the analysis of RFDEs (see [16, 17], for instance), moreover it is also natural from an applied standpoint since random inputs usually have not direct relationship. Hypothesis $\mathbf{H} \mathbf{2}$ is equivalent to assume that $\gamma \in L^{1}(\Omega)$, being $L^{1}(\Omega)$ the biggest of $L^{p}(\Omega)$-Banach spaces [23], so this is a very general assumption since to this space belong the majority of important random variables (Gaussian, Gamma, Beta, etc.). Finally, it is worth mentioning that $\mathbf{H 3}$ only affects the random initial condition $\beta_{0}$ and, again it is fulfilled for most of relevant random variables (Gaussian, Gamma, Beta, etc.,) since it is easy to check that the first derivative of the PDF of random variables is bounded, hence it is Lipschitz.

Let $\varepsilon>0$ arbitrary and let us consider the following development addressed to prove the convergence of $f_{1}^{M}(y, t)$ to $f_{1}(y, t)$ as $M \rightarrow \infty$ for every $(y, t) \in \mathbb{R} \times\left[0, \infty\left[\right.\right.$ fixed, where $t>0$ is such that $(t, \lambda(\omega)) \in \mathcal{N}^{*}(0,0(\omega))$ being $\mathcal{N}^{*}(0,0(\omega))$ 
the neighbourhood whose existence is guaranteed by Remark 1.3

$$
\begin{aligned}
& \left|f_{1}^{M}(y, t)-f_{1}(y, t)\right|=\left|\int_{\mathcal{D}(\gamma)} \int_{\mathcal{D}(\lambda)} f_{\beta_{0}}\left(\frac{y-\gamma S_{2}^{M}(t, \alpha ; \lambda)}{S_{1}^{M}(t, \alpha ; \lambda)}\right) f_{\gamma}(\gamma) f_{\lambda}(\lambda)\right| \frac{1}{S_{1}^{M}(t, \alpha ; \lambda)} \mid \mathrm{d} \lambda \mathrm{d} \gamma \\
& -\int_{\mathcal{D}(\gamma)} \int_{\mathcal{D}(\lambda)} f_{\beta_{0}}\left(\frac{y-\gamma S_{2}(t, \alpha ; \lambda)}{S_{1}(t, \alpha ; \lambda)}\right) f_{\gamma}(\gamma) f_{\lambda}(\lambda)\left|\frac{1}{S_{1}(t, \alpha ; \lambda)}\right| \mathrm{d} \lambda \mathrm{d} \gamma \mid \\
& \leq \int_{\mathcal{D}(\gamma)} \int_{\mathcal{D}(\lambda)}\left|f_{\beta_{0}}\left(\frac{y-\gamma S_{2}^{M}(t, \alpha ; \lambda)}{S_{1}^{M}(t, \alpha ; \lambda)}\right)\right| \frac{1}{S_{1}^{M}(t, \alpha ; \lambda)}\left|-f_{\beta_{0}}\left(\frac{y-\gamma S_{2}^{M}(t, \alpha ; \lambda)}{S_{1}^{M}(t, \alpha ; \lambda)}\right)\right| \frac{1}{S_{1}(t, \alpha ; \lambda)} \mid \\
& +f_{\beta_{0}}\left(\frac{y-\gamma S_{2}^{M}(t, \alpha ; \lambda)}{S_{1}^{M}(t, \alpha ; \lambda)}\right)\left|\frac{1}{S_{1}(t, \alpha ; \lambda)}\right|-f_{\beta_{0}}\left(\frac{y-\gamma S_{2}(t, \alpha ; \lambda)}{S_{1}(t, \alpha ; \lambda)}\right) \mid \frac{1}{S_{1}(t, \alpha ; \lambda)} \| f_{\gamma}(\gamma) f_{\lambda}(\lambda) \mathrm{d} \lambda \mathrm{d} \gamma \\
& \leq \int_{\mathcal{D}(\gamma)} \int_{\mathcal{D}(\lambda)}[\underbrace{f_{\beta_{0}}\left(\frac{y-\gamma S_{2}^{M}(t, \alpha ; \lambda)}{S_{1}^{M}(t, \alpha ; \lambda)}\right)}_{(\mathrm{I})} \underbrace{\left|\frac{1}{S_{1}^{M}(t, \alpha ; \lambda)}\right|-\mid \frac{1}{S_{1}(t, \alpha ; \lambda)} \|}_{(I I)}
\end{aligned}
$$

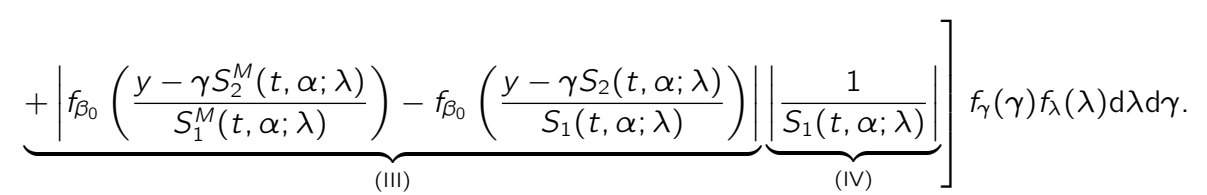

Now, we will obtain some adequate bounds for the terms (I)-(IV) of (14), that will be used later. Let us start with bound (I). According to Remark 1.3, hypothesis $\mathbf{H 3}$, and denoting $F_{0}=f_{\beta_{0}}(0)$ without loss of generality, one obtains

$$
\begin{aligned}
f_{\beta_{0}}\left(\frac{y-\gamma S_{2}^{M}(t, \alpha ; \lambda)}{S_{1}^{M}(t, \alpha ; \lambda)}\right) & \leq\left|f_{\beta_{0}}\left(\frac{y-\gamma S_{2}^{M}(t, \alpha ; \lambda)}{S_{1}^{M}(t, \alpha ; \lambda)}\right)-f_{\beta_{0}}(0)\right|+F_{0} \leq L_{0}\left|\frac{y-\gamma S_{2}^{M}(t, \alpha ; \lambda)}{S_{1}^{M}(t, \alpha ; \lambda)}\right|+F_{0} \\
& \leq L_{0} \frac{|y|+|\gamma|\left|S_{2}^{M}(t, \alpha ; \lambda)\right|}{\left|S_{1}^{M}(t, \alpha, \lambda)\right|}+F_{0} \leq \frac{L_{0}}{m_{S_{1}}}\left(|y|+|\gamma| M_{S_{2}}\right)+F_{0} .
\end{aligned}
$$

Let us obtain a suitable bound for the term (II). On the one hand, by expression (9) of Remark 1.3, we know that $0<m_{S_{1}} \leq\left|S_{1}(t, \alpha ; \lambda)\right|$ for all for all $(t, \lambda) \in \mathcal{N}^{*}(0,0(\omega))$ and $\left.\left.\alpha \in\right] 0,1\right]$. On the other hand, let us recall that in agreement with Remark 1.1 it is known that $S_{i}^{M}(t, \alpha ; \lambda)$, converges uniformly to $S_{i}(t, \alpha ; \lambda)$ for all $(t, \lambda) \in \mathcal{N}^{*}(0,0(\omega))$ and $\left.\left.\alpha \in\right] 0,1\right]$, $i=1$, 2, i.e., for all $\varepsilon_{i}>0$, there exists $M_{0}$, which may depends on $\varepsilon_{i}$, such that

$$
\left|S_{i}(t, \alpha ; \lambda)-S_{i}^{M}(t, \alpha ; \lambda)\right|<\varepsilon_{i}, \quad i=1,2, \quad \forall M \geq M_{0} .
$$

Therefore, applying (16) for $i=1$, one gets

$$
\| \frac{1}{S_{1}^{M}(t, \alpha ; \lambda)}|-| \frac{1}{S_{1}(t, \alpha ; \lambda)}|| \leq\left|\frac{1}{S_{1}^{M}(t, \alpha ; \lambda)}-\frac{1}{S_{1}(t, \alpha ; \lambda)}\right|=\frac{\left|S_{1}(t, \alpha ; \lambda)-S_{1}^{M}(t, \alpha ; \lambda)\right|}{\left|S_{1}^{M}(t, \alpha ; \lambda)\right|\left|S_{1}(t, \alpha ; \lambda)\right|} \leq \frac{\varepsilon_{1}}{\left(m_{S_{1}}\right)^{2}}
$$

As it shall be seen later on, the term (IV) we will be bounded by

$$
\left|1 / S_{1}(t, \alpha ; \lambda)\right|<1 / m_{S_{1}}
$$

which is straightforwardly derived from expression (9) in Remark 1.3. Finally, we will obtain a proper bound to be used for the term (III). To this end, we will apply Remark 1.3, hypothesis $\mathbf{H} 3$ and the convergence of both series $S_{i}(t, \alpha ; \lambda), i=1,2$ (taking 


$$
\begin{aligned}
\varepsilon_{2} & \left.=\frac{\left(m_{S_{1}}\right)^{3}}{L_{0} M_{S_{1}}} \frac{\varepsilon}{2}>\text { in }(16)\right) . \text { This yields } \\
& \left|f_{\beta_{0}}\left(\frac{y-\gamma S_{2}^{M}(t, \alpha ; \lambda)}{S_{1}^{M}(t, \alpha ; \lambda)}\right)-f_{\beta_{0}}\left(\frac{y-\gamma S_{2}(t, \alpha ; \lambda)}{S_{1}(t, \alpha ; \lambda)}\right)\right| \\
& \leq L_{0}\left|\frac{y-\gamma S_{2}^{M}(t, \alpha ; \lambda)}{S_{1}^{M}(t, \alpha ; \lambda)}-\frac{y-\gamma S_{2}(t, \alpha ; \lambda)}{S_{1}(t, \alpha ; \lambda)}\right| \\
& =L_{0} \frac{\left|y S_{1}(t, \alpha ; \lambda)-\gamma S_{2}^{M}(t, \alpha ; \lambda) S_{1}(t, \alpha ; \lambda)-y S_{1}^{M}(t, \alpha ; \lambda)+\gamma S_{2}(t, \alpha ; \lambda) S_{1}^{M}(t, \alpha ; \lambda)\right|}{\left|S_{1}(t, \alpha ; \lambda)\right|\left|S_{1}^{M}(t, \alpha ; \lambda)\right|} \\
& \leq L_{0} \frac{\left[|y|\left|S_{1}(t, \alpha ; \lambda)-S_{1}^{M}(t, \alpha ; \lambda)\right|+|\gamma|\left|S_{2}(t, \alpha ; \lambda) S_{1}^{M}(t, \alpha ; \lambda)-S_{2}^{M}(t, \alpha ; \lambda) S_{1}(t, \alpha ; \lambda)\right|\right]}{\left|S_{1}(t, \alpha ; \lambda)\right|\left|S_{1}^{M}(t, \alpha ; \lambda)\right|} \\
& \leq L_{0} \frac{\left[|y| \varepsilon_{1}+|\gamma|\left|S_{2}(t, \alpha ; \lambda) S_{1}^{M}(t, \alpha ; \lambda)-S_{2}(t, \alpha ; \lambda) S_{1}(t, \alpha ; \lambda)+S_{2}(t, \alpha ; \lambda) S_{1}(t, \alpha ; \lambda)-S_{2}^{M}(t, \alpha ; \lambda) S_{1}(t, \alpha ; \lambda)\right|\right]}{\left|S_{1}(t, \alpha ; \lambda)\right|\left|S_{1}^{M}(t, \alpha ; \lambda)\right|} \\
& \leq L_{0} \frac{\left[|y| \varepsilon_{1}+|\gamma|\left|S_{2}(t, \alpha ; \lambda)\right|\left|S_{1}^{M}(t, \alpha ; \lambda)-S_{1}(t, \alpha ; \lambda)\right|+\left|S_{1}(t, \alpha ; \lambda)\right|\left|S_{2}(t, \alpha ; \lambda)-S_{2}^{M}(t, \alpha ; \lambda)\right|\right]}{\left|S_{1}(t, \alpha ; \lambda)\right|\left|S_{1}^{M}(t, \alpha ; \lambda)\right|} \\
& \leq \frac{L_{0}}{\left(m_{S_{1}}\right)^{2}}\left[|y| \varepsilon_{1}+|\gamma| M S_{2} \varepsilon_{1}+M_{S_{1}} \varepsilon_{2}\right]=\frac{L_{0}}{\left(m_{S_{1}}\right)^{2}}\left(|y|+|\gamma| M S_{2}\right) \varepsilon_{1}+\frac{m_{S_{1}} \varepsilon}{2} .
\end{aligned}
$$

Now, we firstly substitute bounds (15), (17)-(19) in (14), secondly we apply that $\int_{\mathcal{D}(\lambda)} f_{\lambda}(\lambda) \mathrm{d} \lambda=1$ and the expression of expectation operator via an integral as well. This leads

$$
\begin{aligned}
\left|f_{1}^{M}(y, t)-f_{1}(y, t)\right| \leq & \int_{\mathcal{D}(\gamma)} \int_{\mathcal{D}(\lambda)}\left[\left(\frac{L_{0}}{m_{S_{1}}}\left(|y|+|\gamma| M_{S_{2}}\right)+F_{0}\right) \frac{\varepsilon_{1}}{\left(m_{S_{1}}\right)^{2}}\right. \\
& \left.+\left(\frac{L_{0}}{\left(m_{S_{1}}\right)^{2}}\left(|y|+|\gamma| M_{S_{2}}\right) \varepsilon_{1}+\frac{m_{S_{1}} \varepsilon}{2}\right) \frac{1}{m_{S_{1}}}\right] f_{\gamma}(\gamma) f_{\lambda}(\lambda) \mathrm{d} \lambda \mathrm{d} \gamma \\
= & \int_{\mathcal{D}(\gamma)}\left[\left(\frac{2 L_{0}}{\left(m_{S_{1}}\right)^{3}}\left(|y|+|\gamma| M_{S_{2}}\right)+\frac{F_{0}}{\left(m_{S_{1}}\right)^{2}}\right) \varepsilon_{1}+\frac{\varepsilon}{2}\right] f_{\gamma}(\gamma) \mathrm{d} \gamma \\
= & \mathbb{E}\left[\left(\frac{2 L_{0}}{\left(m_{S_{1}}\right)^{3}}\left(|y|+|\gamma| M_{S_{2}}\right)+\frac{F_{0}}{\left(m_{S_{1}}\right)^{2}}\right) \varepsilon_{1}+\frac{\varepsilon}{2}\right] \\
= & \left(\frac{2 L_{0}}{\left(m_{\left.S_{1}\right)^{3}}\left(|y|+\mathbb{E}[|\gamma|] M_{S_{2}}\right)+\frac{F_{0}}{\left(m_{S_{1}}\right)^{2}}\right) \varepsilon_{1}+\frac{\varepsilon}{2} .}\right.
\end{aligned}
$$

Finally, taking $\varepsilon_{1}=\frac{\left(m_{S_{1}}\right)^{3}}{2 L_{0}\left(|y|+C M_{S_{2}}\right)+F_{0} m_{S_{1}}} \frac{\varepsilon}{2}$ and applying hypothesis $\mathbf{H} \mathbf{2}$ in the latter expression, one concludes

$$
\left|f_{1}^{M}(y, t)-f_{1}(y, t)\right| \leq\left(\frac{2 L_{0}}{\left(m_{S_{1}}\right)^{3}}\left(|y|+C M_{S_{2}}\right)+\frac{F_{0}}{\left(m_{S_{1}}\right)^{2}}\right) \varepsilon_{1}+\frac{\varepsilon}{2}=\frac{\varepsilon}{2}+\frac{\varepsilon}{2}=\varepsilon .
$$

Summarizing the following result has been established:

Theorem 2.1 Let us consider random IVP (2) and assume that

H1: $\beta_{0}(\omega), \gamma(\omega)$ and $\lambda(\omega)$ are independent random variables defined in a common complete probability space $(\Omega, \mathcal{F}, \mathbb{P})$.

H2: $\mathbb{E}[|\gamma(\omega)|]=C<\infty$.

H3: The PDF $f_{\beta_{0}}\left(\beta_{0}\right)$ of $\beta_{0}(\omega)$ is Lipschitz continuous in $\mathbb{R}$.

Let $S_{1}^{M}(t, \alpha ; \lambda)$ and $S_{2}^{M}(t, \alpha ; \lambda)$ denote the random finite sums given by (6). Then, $f_{1}^{M}(y, t)$ defined by $(13)$ is the first probability density function of the approximate solution stochastic process (5) of random IVP (2). Furthermore, for each $(y, t) \in \mathbb{R} \times] 0, \infty[$ fixed, $f_{1}^{M}(y, t)$ converges to the first probability density function $f_{1}(y, t)$, given by $(12)$, of the exact solution $y(t, \omega)$ defined by (3) $-(4)$.

\section{Numerical examples}

In this section we will show two numerical examples addressed to illustrate our previous theoretical findings. In each example, a variety of probability distributions for input parameters of random fractional IVP (2) will be considered. 

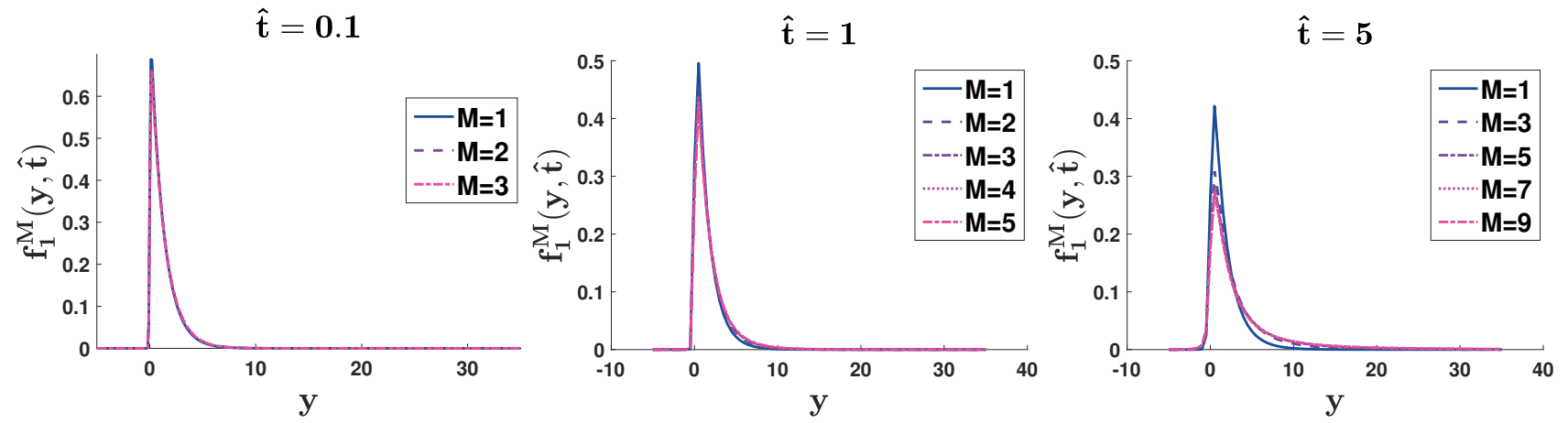

Figure 1. Plots of the 1-PDF, $f_{1}^{M}(y, t)$, given by (13), for different truncations $M$ at the time instants $\hat{t}=0.1$ (left), $\hat{t}=1$ (center) and $\hat{t}=5$ (right) in Example 1.

\begin{tabular}{lcccccccc}
\hline$e_{M}(\hat{t})$ & $\mathrm{M}=1$ & $\mathrm{M}=2$ & $\mathrm{M}=3$ & $\mathrm{M}=4$ & $\mathrm{M}=5$ & $\mathrm{M}=6$ & $\mathrm{M}=7$ & $\mathrm{M}=8$ \\
\hline \hline$\hat{t}=0.1$ & 0.031 & 0.006 & - & - & - & - & - & - \\
\hline$\hat{t}=1$ & 0.094 & 0.040 & 0.019 & 0.009 & - & - & - & - \\
\hline$\hat{t}=5$ & 0.190 & 0.105 & 0.064 & 0.041 & 0.027 & 0.018 & 0.013 & 0.009 \\
\hline
\end{tabular}

Table 1. Values of error $e_{M}(\hat{t})$ defined in (20) at the time instants $\hat{t}=\{0.1,1,5\}$ in Example 1 . The truncation order $M$ has been computed so that $e_{M}(\hat{t}) \leq 10^{-2}$.

\subsection{Example 1}

Let us assume that the fractional order of Caputo derivative is $\alpha=0.3$, the diffusion coefficient $\lambda(\omega)$ has a Beta distribution with parameters 30 and 40 , i.e., $\lambda(\omega) \sim \operatorname{Be}(30 ; 40)$, the non-homogeneous term $\gamma(\omega)$ is a Gaussian RV with mean 0 and standard deviation $0.1, \gamma(\omega) \sim N(0 ; 0.1)$ and the initial condition $\beta_{0}(\omega)$ has an Exponential distribution with mean $1, \beta_{0}(\omega) \sim \operatorname{Exp}(1)$. In agreement with hypothesis $\mathbf{H 1}$, we will assume that $\lambda(\omega), \gamma(\omega)$ and $\beta_{0}(\omega)$ are independent. A direct computation shows that $\mathbb{E}[|\gamma|]=\frac{1}{5 \sqrt{2 \pi}}<\infty$, thus hypothesis $\mathbf{H} 2$ holds. Also the PDF of $\beta_{0}(\omega)$ is $f_{\beta_{0}}\left(\beta_{0}\right)=\mathrm{e}^{-\beta_{0}}, \beta_{0}>0$ (and null otherwise), it first derivative satisfies $\left|f_{\beta_{0}}^{\prime}\left(\beta_{0}\right)\right|=e^{-\beta_{0}}<1$ for all $\beta_{0}>0$, i.e., $f_{\beta_{0}}$ is bounded and hence is Lipschitz. As a result, hypothesis $\mathbf{H 3}$ fulfils too.

In Figure 1 the approximate 1-PDF, $f_{1}^{M}(y, t)$, given by (13) has been plotted for different values of the truncation order $M$ and the time instants $\hat{t}=\{0.1,1,5\}$. From it, one can observe that rapid convergence of approximations. As a measure of the error of these approximations, we have define

$$
e_{M}(\hat{t})=\int_{-\infty}^{\infty}\left|f_{1}^{M}(y, \hat{t})-f_{1}^{M+1}(y, \hat{t})\right| d y, \quad M \geq 1 \text { integer }
$$

In Table 1 , we have collected these errors for time instants $\hat{t}=\{0.1,1,5\}$ taking as stopping criterion that $e_{M}(\hat{t}) \leq 10^{-2}$.

In Figure 2, we show the mean, $\mu_{y M}(t)$, and the variance, $\sigma_{y M}^{2}(t)$, for different values of truncation order $M$ on the time interval $t \in[0,5]$. In these plots, the convergence of both statistical moments is clearly observed. To account for the quality of approximations of the mean and the variance, we consider the following global error over the whole time interval $[0,5]$

$$
e_{M}^{\mu}=\int_{0}^{5}\left|\mu_{y^{M}}(t)-\mu_{y^{M+1}}(t)\right| \mathrm{d} t, \quad e_{M}^{\sigma^{2}}=\int_{0}^{5}\left|\sigma_{y^{M}}^{2}(t)-\sigma_{y^{M+1}}^{2}(t)\right| \mathrm{d} t, \quad M \geq 1 \text { integer. }
$$

In agreement with (1), the above term $\mu_{y^{M}}(t)$ is computed as

$$
\mu_{y^{M}}(t)=\mathbb{E}\left[y^{M}(t, \omega)\right]=\int_{-\infty}^{\infty} y^{M}(t) f_{1}^{M}(y, t) \mathrm{d} y, \quad \omega \in \Omega
$$

where $y^{M}(t, \omega)$ and $f_{1}^{M}(y, t)$ are given by, (5)-(6) and (13), respectively. Analogously,

$$
\sigma_{y^{M}}^{2}(t)=\mathbb{E}\left[\left(y^{M}(t, \omega)\right)^{2}\right]-\left(\mathbb{E}\left[y^{M}(t, \omega)\right]\right)^{2}=\int_{-\infty}^{\infty}\left(y^{M}(t)\right)^{2} f_{1}^{M}(y, t) \mathrm{d} y-\left(\mu_{y^{M}}(t)\right)^{2}, \quad \omega \in \Omega .
$$

In Table 2, we show the values of errors defined in (21). These figures have been computed taking values of the truncation order $M$ so that error is less or equal than $10^{-2}$. Notice that greater values of $M$ are required to accomplish for the stopping criterion of the error of the variance as expected. From this table we observe that both errors, $e_{M}^{\mu}$ and $e_{M}^{\sigma^{2}}$, decrease as $M$ increases. 

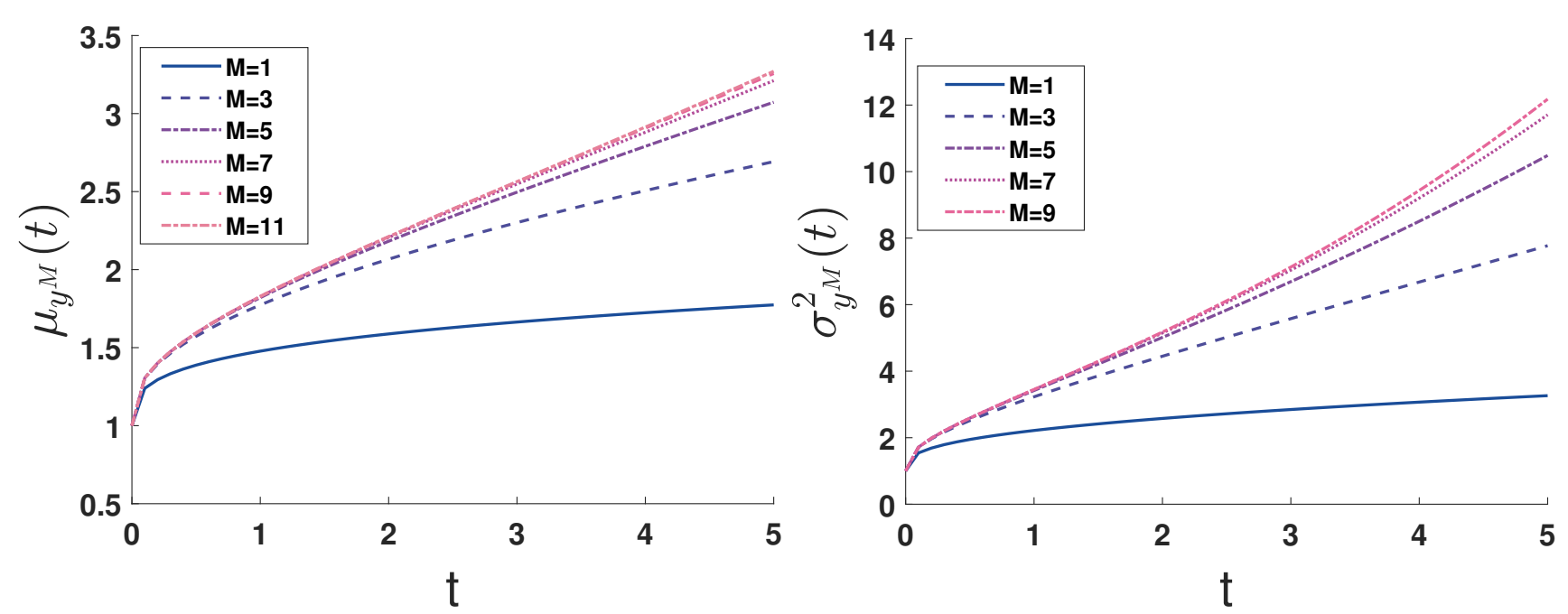

Figure 2. Plots of approximations $\mu_{y} M(t)$ (left) and $\sigma_{y M}^{2}(t)$ (right), given by (22) and (23), respectively, to the exact mean and variance of the solution stochastic process to random fractional IVP (2), respectively, for different values of the truncation order $M$ in the context of Example 1.

\begin{tabular}{|c|c|c|c|c|c|c|c|}
\hline & $M=1$ & $M=2$ & $M=3$ & $M=4$ & $M=5$ & $M=6$ & $M=7$ \\
\hline$e_{M}^{\mu}$ & 1.746 & 0.987 & 0.547 & 0.298 & 0.160 & 0.085 & 0.045 \\
\hline \multirow[t]{2}{*}{$e_{M}^{\sigma^{2}}$} & 6.877 & 4.871 & 3.164 & 1.949 & 1.157 & 0.668 & 0.378 \\
\hline & $M=8$ & $M=9$ & $M=10$ & $M=$ & & & $M=13$ \\
\hline$e_{M}^{\mu}$ & 0.023 & 0.012 & 0.006 & - & & - & - \\
\hline$e_{M}^{\sigma^{2}}$ & 0.210 & 0.115 & 0.062 & 0.03 & & 178 & 0.0094 \\
\hline
\end{tabular}

Table 2. Values of errors defined in (21) to quantify the quality of approximations $\mu_{y M}(t)$ and $\sigma_{y^{M}}^{2}(t)$, given by (22) and (23), respectively, to the exact mean and variance of the solution stochastic process to random fractional IVP (2), respectively, for different values of the truncation order $M$ in the context of Example 1 . The truncation order $M$ has been computed so that $e_{M}(\hat{t}) \leq 10^{-2}$.

\begin{tabular}{lcccccccccc}
\hline $\mathrm{e}_{M}(\hat{t})$ & $\mathrm{M}=1$ & $\mathrm{M}=2$ & $\mathrm{M}=3$ & $\mathrm{M}=4$ & $\mathrm{M}=5$ & $\mathrm{M}=6$ & $\mathrm{M}=7$ & $\mathrm{M}=8$ & $\mathrm{M}=9$ & $\mathrm{M}=10$ \\
\hline \hline$\hat{t}=0.05$ & 0.009 & - & - & - & - & - & - & - & - & - \\
\hline$\hat{t}=0.2$ & 0.338 & 0.159 & 0.077 & 0.038 & 0.018 & 0.009 & - & - & - & - \\
\hline$\hat{t}=1$ & 0.817 & 0.516 & 0.337 & 0.223 & 0.148 & 0.099 & 0.066 & 0.043 & 0.029 & 0.010 \\
\hline
\end{tabular}

Table 3. Values of error $e_{M}(\hat{t})$ defined in (20) at the time instants $\hat{t}=\{0.05,0.2,1\}$ in Example 2. The truncation order $M$ has been computed so that $e_{M}(\hat{t}) \leq 10^{-2}$.

\subsection{Example 2}

This second example aims to complete our numerical experiments by taking different probability distributions for random inputs and the order of fractional derivative from the ones chosen in Example 1. In this spirit, our subsequent presentation will be more concise since it follows in broad outline a similar structure as in Example 1. Let us consider the random fractional IVP (2) where $\alpha=0.7, \lambda(\omega)$ is a Gaussian random variable with mean 1 and standard deviation $0.1, \lambda(\omega) \sim N(1 ; 0.1)$, $\gamma(\omega)$ has a Uniform distribution on interval [2,3], $\gamma(\omega) \sim \operatorname{Un}(2,3)$ and $\beta_{0}(\omega)$ has a Gamma distribution with parameters 1 and $2, \beta_{0} \sim \mathrm{Ga}(1 ; 2)$. Hereinafter, we assume that $\lambda(\omega), \gamma(\omega)$ and $\beta_{0}(\omega)$ are independent, this ensures hypothesis $\mathbf{H} \mathbf{1}$ is fulfilled. Furthermore, it is clear that $\mathbb{E}[|\gamma|]=5 / 2$ and $\left|f_{\beta_{0}}^{\prime}\left(\beta_{0}\right)\right|=\left|\left(1 / 2 e^{-\beta_{0} / 2}\right)^{\prime}\right| \leq 1 / 4$ for all $\beta_{0}>0$. As a consequence, hypotheses $\mathbf{H} 2$ and $\mathbf{H} \mathbf{3}$ hold.

In Figure 3, we have plotted the 1-PDF, $f_{1}^{M}(y, t)$ given in (13), for different orders of truncation $M$ and time instants $\hat{t}=\{0.05,0.2,1\}$. From these graphical representations, we can observe the convergence of approximations even using few terms. As a measure of the accuracy of these approximations, in Table 3 we show the values of error defined in (20) time instants $\hat{t}=\{0.05,0.2,1\}$ taking as stopping criterion for the order of truncation $M$ that $e_{M}(\hat{t}) \leq 10^{-2}$.

Finally, in Figure 4 the mean, $\mu_{y M}(t)$ and the variance $\sigma_{y M}^{2}(t)$ for different truncation order $M$ are plotted to $t$ from 0 to 1 . As in the case of the 1-PDF we can observe the convergence. In Table 4 the following error for the mean and the variance is calculated as in Example 3.1. 

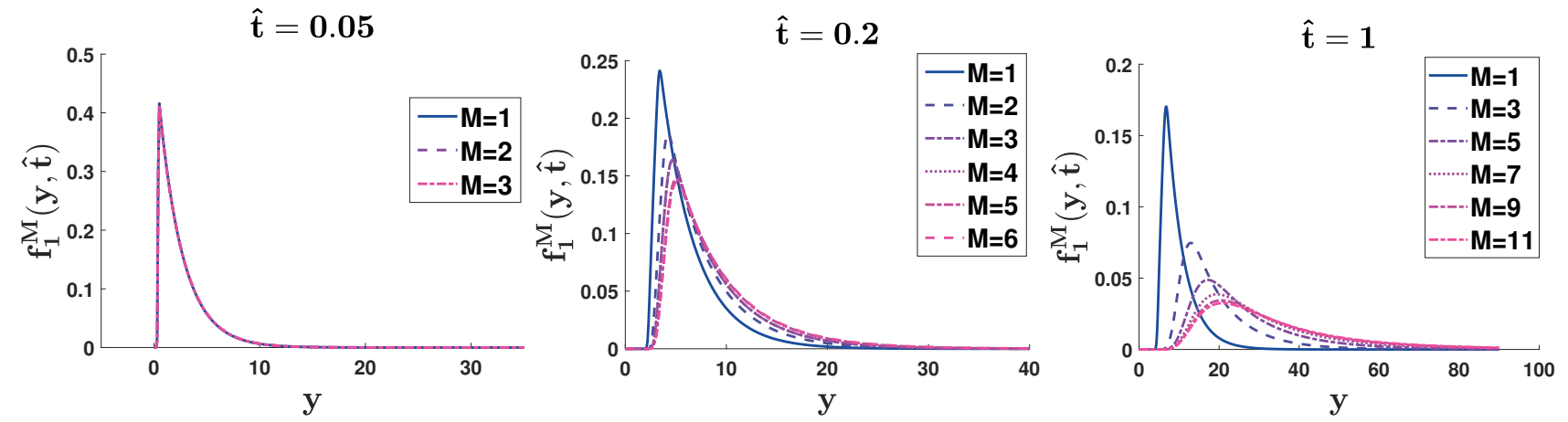

Figure 3. Plots of the 1-PDF, $f_{1}^{M}(y, t)$, given by (13), for different truncations $M$ at the time instants $\hat{t}=0.05$ (left), $\hat{t}=0.2$ (center) and $\hat{t}=1$ (right) in Example 2.
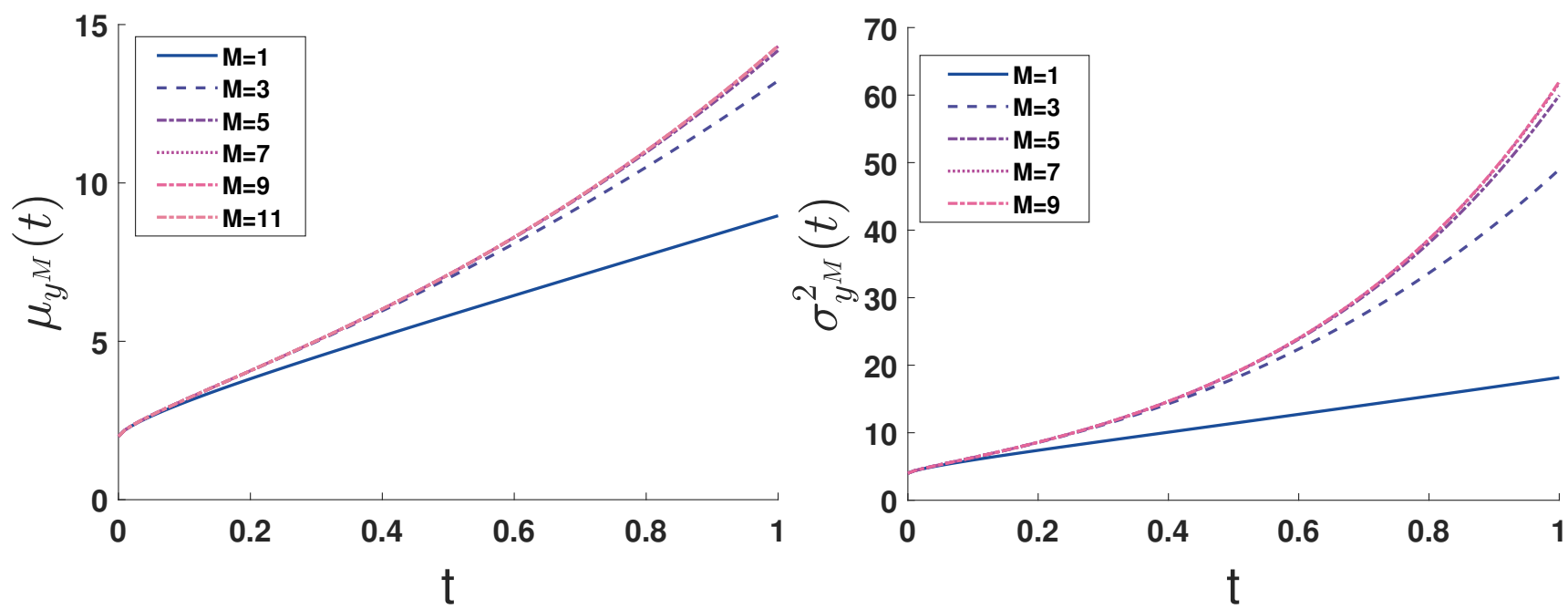

Figure 4. Plots of approximations $\mu_{y^{M}}(t)$ (left) and $\sigma_{y M}^{2}(t)$ (right), given by (22) and (23), respectively, to the exact mean and variance of the solution stochastic process to random fractional IVP (2), respectively, for different values of the truncation order $M$ in the context of Example 2.

\begin{tabular}{ccccccc}
\hline & $M=1$ & $M=2$ & $M=3$ & $M=4$ & $M=5$ & $M=6$ \\
\hline \hline$e_{M}^{\mu}$ & 1.062 & 0.454 & 0.173 & 0.060 & 0.019 & 0.006 \\
\hline$e_{M}^{\sigma^{2}}$ & 0.712 & 0.331 & 0.136 & 0.050 & 0.017 & 0.005 \\
\hline
\end{tabular}

Table 4. Values of errors defined in (21) to quantify the quality of approximations $\mu_{y M}(t)$ and $\sigma_{y^{M}}^{2}(t)$, given by (22) and (23), respectively, to the exact mean and variance of the solution stochastic process to random fractional IVP (2), respectively, for different values of the truncation order $M$ in the context of Example 2. The truncation order $M$ has been computed so that

$$
e_{M}(\hat{t}) \leq 10^{-2} \text {. }
$$

\section{Conclusions}

In this paper, we have computed approximations to the first probability density function of random fractional linear differential equations using the so-called Random Variable Transformation method. Under mild conditions on random data, we have proved the convergence of approximations to the exact probability density function of the solution stochastic process. The generality of our findings relies upon the facts that uncertainty has been considered in all inputs (initial condition, forcing term and diffusion coefficient) and a wide variety of probability distributions can be assigned to each one of them. To the best of our knowledge, this is the first time this analysis has been conducted for random fractional differential equations. We hope this contribution stimulate further studies in the emergent realm of Fractional Calculus with randomness. 


\section{Acknowledgements}

This work has been partially supported by the Ministerio de Economía y Competitividad grant MTM2017-89664-P. Ana Navarro Quiles acknowledges the doctorate scholarship granted by Programa de Ayudas de Investigación y Desarrollo (PAID-2014), Universitat Politècnica de València.

\section{Conflict of Interest Statement}

The authors declare that there is no conflict of interests regarding the publication of this article.

\section{References}

1. Samko SG, Kilbas AA, Marichev OI. Fractional integrals and derivatives, theory and applications. CRC Press, 1993.

2. Kilbas AA, Srivastava HM, Trujillo JJ. Theory and applications of fractional differential equations. Elsevier Science, 2006.

3. Bouras Y, Zorica F, Atanacković TM, Vrcelj Z. A non-linear thermo-viscoelastic rheological model based on fractional derivatives for high temperature creep in concrete. Applied Mathematical Modelling 2018; 55:551-568.

4. Tarasov VE. Fractional integro-differential equations for electromagnetic waves in dielectric media. Theoretical and Mathematical Physics 2009;158:355-359.

5. Acedo L, Burgos C, Cortés J-C, Villanueva R-J. Probabilistic prediction of outbreaks of meningococcus W-135 infections over the next few years in Spain. Physica A 2017; 486:106-117.

6. Agarwal RP, Lakshmikantham V, Nieto JJ. On the concept of solution for fractional differential equations with uncertainty. Nonlinear Analysis: Theory, Methods \& Applications 2010;72:2859-2862.

7. Pedjeu J-C, Ladde GS. Stochastic fractional differential equations: Modeling, method and analysis. Chaos, Solitons \& Fractals 2012; 45(3):279-293.

8. Oksendal B. Stochastic Differential Equations. An Introduction with Applications. Springer-Verlag Berlin Heidelberg, 1998

9. Kloeden E, Platen E. Numerical Solution of Stochastic Differential Equations. Springer-Verlag Berlin Heidelberg, 1992.

10. Debbouche A, Nieto J. Sobolev type fractional abstract evolution equations with nonlocal conditions and optimal multi-controls. Applied Mathematics and Computation 2014; 245:74-85.

11. Mourad K, Debbouche A. Complete controllability of nonlocal fractional stochastic differential evolution equations with Poisson jumps in Hilbert spaces. International Journal of Advances in Applied Mathematics and Mechanics 2015; 3(1):41-48.

12. Zhu Y. Uncertain fractional differential equations and interest rate model. Mathematical Methods in the Applied Sciences 2015;38:3359-3368.

13. Lupulescu V, Ntouyas KN. Random fractional differential equations. International Electronic Journal of Pure and Applied Mathematics 2012; 4(2):119-136.

14. Lupulescu V, OReagan D, Rahman GU. Existence results for random fractional differential equations. Opuscula Mathematica 2014; 34(4):813-825.

15. González-Parra G, Chen-Charpentier BM, Arenas AJ. Polynomial chaos for randomfractional order differential equations. Applied Mathematics and Computation 2014; 226:123-130.

16. Burgos C, Cortés J-C, Villafuerte L, Villanueva R-J. Extending the deterministic Riemann-Liouville and Caputo operators to the random framework: A mean square approach with applications to solve random fractional differential equations. Chaos, Solitons \& Fractals 2017; 102:305-318.

17. Burgos C, Calatayud J, Cortés J-C, Villafuerte L. Solving a class of random non-autonomous linear fractional differential equations by means of a generalized mean square convergent power series. Applied Mathematics Letters 2018; 78:95-104.

18. Dorini FA, Cecconello MS, Dorini LB. On the logistic equation subject to uncertainties in the environmental carrying capacity and initial population density. Communications in Nonlinear Science and Numerical Simulation 2016; 33:160-173.

19. Hussein A., Selim M.M. Solution of the stochastic radiative transfer equation with Rayleigh scattering using RVT technique. Applied Mathematics and Computation 2012; 218(13):7193-7203.

20. Casabán M-C, Cortés J-C, Navarro-Quiles A, Romero J-V, Roselló M-D, Villanueva R-J. A comprehensive probabilistic solution of random SIS-type epidemiological models using the random variable transformation technique. Communications in Nonlinear Science and Numerical Simulation 2016; 32:199-210.

21. Casabán M-C, Cortés J-C, Romero J-V, Roselló M-D. Solving random homogeneous linear second-order differential equations: A full probabilistic description. Mediterranean Journal of Mathematics 2016; 13(6):3817-3836.

22. Soong TT. Random Differential Equations in Science and Engineering. Academic Press New York, 1973.

23. Loève M. Probability Theory. Vols. I-II. Springer-Verlag New York, 1977. 